\title{
An X-linked dominant mutation in LAMP2 causing Danon disease associated with myotonia expanding the spectrum
}

S.H.S. Chan ${ }^{1}$, A. Kan ${ }^{2}$, H.F. Tse ${ }^{3}$

${ }^{1}$ Depratment of Paediatrics and Adolescent Medicine, Queen Mary Hospital, Hong Kong;

${ }^{2}$ Department of Pathology, Queen Mary Hospital, Hong Kong; ${ }^{3}$ Department of Medicine, Queen Mary Hospital, Hong Kong

We describe a family with strong family history of cardiomyopathy. The mother has dilated cardiomyopathy with symptoms onset around 40 years old requiring treatment for both heart failure and atrial fibrillation. She does not have muscle weakness and her creatine kinase level was normal. The elder son has hypertrophic cardiomyopathy with symptoms onset at 20 years old, a mildly elevated creatine kinase of $1000 \mathrm{U} / \mathrm{L}$ but no muscle weakness. The younger brother with limited intelligence was asymptomatic all along. At the age of 15 with an incidental finding of raised serum transaminase levels he was referred for further investigations. Initial consultation confirmed mild proximal weakness, calves hypertrophy, creatine kinase up to $3500 \mathrm{U} / \mathrm{L}$ and echocardiogram confirmed hypertrophic cardiomyopathy. Needle electromyography showed myotonia after ice application. Nerve conduction study with short exercise test showed compound muscle action potentials decrement confirming myotonia. Metabolic workup, DMD gene and CTG repeat mutation study were all negative. The muscle biopsy of the elder brother showed vacuolar myopathy. Subsequent LAMP2 mutation study on exome sequencing confirmed Danon Disease. Mother has subsequently undergone heart transplantation, and vacuolar myocytes are noted in the cardiac muscles. This report describe a family with Danon disease, a rare X-linked dominant condition, but an important differential diagnosis for patient with cardiomyopathy, myopathy and learning difficulty, having a strong family history of cardiomyopathy. While skeletal muscles biopsy showed vacuolar myopathy, these abnormal vacuolar changes also extended to the cardiac muscles. Diagnostic confirmation is important when counseling the long term prognosis for this family. While symptomatic treatment is available for the heart failure, with a progressive course of cardiomyopathy the ultimate cure is heart transplantation. 